\title{
Интегральный показатель экономической добавленной стоимости: проблема учета стоимости интеллектуального капитала компании
}

\author{
Исакин М.А. ${ }^{22}$, Осколкова М.А. ${ }^{23}$, Шакина Е.А. ${ }^{24}$
}

Определяя стратегические ориентиры развития компании, менеджеры и собственники практически всегда испытывают дефиџит информаџии о том, какой потенциал эффективности имеет каждый из объектов инвестиций. В самом первом приближении компания должна найти компромисс между инвестициями в традиционные материальные активы и формированием интеллектуального капитала. Конщепџия RAVE исходит преимущественно из предпосылки о том, что в условиях новой экономки на первый план выходит не основной капитал, а интеллектуальный, в том числе знания, которыми обладает и которые использует компания (в том числе в сфере маркетинга, снабжения и развития персонала). Настоящая статья посвящена вопросам развития разработанного консультантами ВCG подхода, в частности обоснованию метода оценки интегрального показателя экономической добавленной стоимости.

\section{JEL: $G 30$}

Ключевые слова: интеллектуальный капитал, экономическая добавленная стоимость, эффективность деятельность компании.

Концепции финансового менеджмента за последние десятилетия сменяли одна другую, критикуя нецелостность предыдущих. Так, концепция стремления к росту выручки критиковалась в связи с необходимостью оптимизации затрат компании и, как следствие, стремлением к максимизации прибыли. Последняя, в свою очередь, была признана несостоятельной в долгосрочной перспективе и сменилась стоимостной концепцией, которая по сей день рассматривается как наиболее точная целевая установка рациональных инвесторов компании, так как отражает мотивы аккумулирования капитала для осуществления предпринимательской деятельности. Несмотря на это, в современных источниках все чаще можно встретить попытку критики концепции максимизации стоимости, обоснованную преимущественно фактическим разрывом теоретического подхода и реальной практики ведения бизнеса, что, в сущности, характеризует нерациональность конкретных инвесторов. Очевидно, что говоря об эффективности деятельности компании, исследователи, практикующие аналитики и менеджеры компании могут подразумевать различные критерии ее достижения. Мы, тем не менее, должны в качестве основы построения теоретических гипотез о поведении фирмы определить концепцию, которая при прочих равных условиях отражает мейнстрим в целевых установках экономических агентов. При этом необходимо учитывать постоянно меняющуюся внешнюю среду рынков, на которых действуют эти агенты, а именно переход экономики в целом на новый уровень развития. В настоящее время можно уже без сомнений говорить, по крайней мере, о начальном этапе развития экономики знаний (отметим, что это не исключает высокую роль традиционных ресурсов фирмы). Возросшая в последнее время роль интеллектуального капитала формирует принципиально новую сложную сетевую структуру экономики, где ни один из стейкхолдеров не может быть признан ключевым. В отличие от финансового капитала, обеспеченного инвесторами компании, поставщиками интеллектуального капитала

\footnotetext{
${ }^{22}$ Доцент кафедры финансового менеджмента ПФ ГУ ВШЭ, канд. эконом. наук.

${ }_{23}$ Преподаватель кафедры финансового менеджмента ПФ ГУ ВШЭ.

${ }^{24}$ Доцент кафедры финансового менеджмента ПФ ГУ ВШЭ, канд .эконом. наук.
} 
являются работники и менеджеры компании, все ее контрагенты, а также представители инфраструктуры, без которой не может функционировать фирма.

При этом концепция стратегического управления компании, основанная на создании стоимости, базируется в первую очередь на предпосылке о том, что любой собственник заинтересован в росте стоимости своих активов. Однако очевидным является то, что выгоды от подобного роста получают все ключевые стейкхолдеры компании: государство, работники, менеджеры, потребители и поставщики. Рост эффективности компании приводит к увеличению налоговых платежей в бюджет, обеспечивает стабильную заработную плату и гарантии для занятых в ее деятельности людей; для потребителей созданная компанией стоимость обеспечивает высокое качество продукции по справедливой цене; поставщики получают рынки сбыта и т.д.

Определяя стратегические ориентиры развития компании, менеджеры и собственники практически всегда испытывают дефицит информации о том, какой потенциал эффективности имеет каждый из объектов инвестиций. По мнению авторов, компания должна найти компромисс между инвестициями в традиционные материальные активы и формированием интеллектуального капитала. Ориентируясь на опыт прошлого и в связи с тем, что отдача от материальных активов часто является более очевидной, руководство компании принимает в большинстве случаев решение о расширении деятельности за счет накопления материального капитала (оборудования, недвижимости, запасов и т.д.). Исключение представляют лишь те компании, где материальные активы не создают основу деятельности (к ним относятся преимущественно фирмы третичного сектора, в том числе инжиниринговые и консалтинговые компании, юридические и кадровые агентства и т.д.). Однако в условиях так называемой «новой экономики» компании всех отраслей будут конкурировать именно за эффективность использования неосязаемых активов, в частности, интеллектуального капитала, так как данные факторы сегодня определяют основные рыночные преимущества практически любого экономического агента.

Так, успех компании в большей степени обусловлен нестандартными маркетинговыми решениями, лояльностью покупателей и клиентов, способностью быстро и гибко реагировать на изменение внешней среды, нежели объемом и стоимостью физического и финансового капитала. Таким образом, для описанных условий должны появляться принципиально новые методы оценки добавленной стоимости компании.

Финансовые индикаторы оценивают лишь те аспекты деятельности компании, которые связаны с инвестициями в материальные активы: технологическое оборудование, материальные запасы и т.д. При этом за кадром остаются все ключевые элементы интеллектуального капитала, в том числе человеческие ресурсы, опыт и репутация компании на рынке, а также долгосрочные контракты с поставщиками и покупателями. Финансовый капитал действительно играет ключевую роль в крупных корпорациях, привлекающих большую часть финансирования на фондовом рынке. В этом случае практически реализуется предпосылка о том, что в стоимости привлеченного капитала отражены все основные операционные и финансовые риски, обоснованные, в том числе, использованием интеллектуального капитала. Тем не менее даже в случае анализа открытой корпорации оценка будет смещена за счет нефинансовых факторов успешного функционирования компании, неучтенных фондовым рынком. Оценивая стоимость закрытой компании, мы сталкиваемся с тем, что попытки оценить добавленную стоимость ее капитала через исключительно финансовые результаты ее деятельности могут привести к существенно искаженным выводам.

При любом из вариантов оценки стоимости компании в целях определения того, создает ли объект анализа добавочную стоимость, мы отвечаем на следующий вопрос: «могут ли ресурсы, вложенные в компанию, приносить большую эффективность по сравнению с альтернативными вариантами их использования?». Так, в терминах материальных активов, это утверждение может быть проинтерпретировано следующим образом: приносит ли вложенный в компанию финансовый капитал доходность выше, чем 
величина затрат, связанная с его привлечением. В терминах неосязаемых активов компания, которая создает добавочную стоимость, привлекает лучших работников, формирует лояльность, генерирует больше денежных потоков, а также дает некую гарантию стабильности, что позволяет менеджменту принимать решения на долгосрочный период.

Таким образом, в том, что компания выигрывает от создания добавочной стоимости, нет сомнений, однако для того, чтобы определить эту возможность, необходимо уметь оценить ее, измерить в тех или иных показателях. На сегодняшний день существует множество измерителей добавленной стоимости компании, которые призваны выступать инструментами оценки при принятии стратегических решений в компании. Однако каждый из них имеет ряд ограничений, среди которых обычно выделяют:

- отсутствие универсальных методов расчета;

- дефицит информационной базы для оценки;

- неоднозначность интерпретации результатов;

- учет преимущественно материальных активов и отдачи от их использования.

Появление новых методов оценки добавленной стоимости, в сущности, обосновано наличием перечисленных недостатков существующих инструментов. При этом на сегодняшний день можно говорить о том, что проблемы универсальной информационной базы и алгоритма оценки решает развитие метода Стерна-Стюарда, основанного на получении финансовых показателей из учетных посредством ряда обоснованных корректировок, входящих в расчет экономической добавленной стоимости показателей. Степень неоднозначности интерпретации результатов оценки снижает показатель денежной добавленной стоимости, который в большей степени отражает суть универсального метода оценки стоимости компании, основанного на подходе DCF. В связи с этим самыми популярными на сегодняшний день являются концепции CVA и EVA, которые используют уже более 250 компаний с мировым именем (например, AT\&T, Quarker Oats, Briggs\&Stratton, Coca-Cola [Редченко, 2002]), а также множество средних и мелких фирм.

На протяжении десятилетий EVA была очень популярна как на Западе, так и в России. Многие финансовые менеджеры считали, что они находят правильные ответы, измеряя деятельность фирмы с помощью этого показателя.

В 2001 году испанский аналитик П. Фернандес проанализировал информацию, предоставленную компанией Stern Stewart о 582 американских компаниях, использующих EVA, MVA, NOPAT и WACC. На основании корреляционного анализа он сделал вывод, что EVA и CVA не способны измерить создание стоимости компанией [Fernandez, 2001]. В своем анализе он использовал внешний способ измерения, если говорить в терминологии BCG, используя как ключевой показатель добавленную рыночную стоимость компании.

Для 210 компаний корреляция добавленной рыночной стоимости с показателем EVA была отрицательной. Средняя корреляция между ростом добавленной рыночной стоимости и EVA составила 16\%. Корреляция между доходом собственников и ростом CVA для 100 наиболее прибыльных мировых компаний составила 1,7\%.

Таким образом, это исследование опровергает рекламный слоган компании Stern\&Stewart, говорящий о том, что рост показателя EVA является ключевым для роста создаваемой компанией стоимости [Ehrbar, 1998].

То есть для описанных выше условий должны появляться принципиально новые методы оценки добавленной стоимости компании. Первым шагом к изменению концепций в сфере стратегического управления стало появление концепции сбалансированных показателей Нортана-Каплана. Balanced Scorecard разработана на основе выводов исследования, проведенного в 1990 году профессорами Гарвардской школы экономики Дэвидом Нортоном и Робертом Капланом. Исследование проводилось с целью выявления новых способов повышения эффективности деятельности и достижения целей бизнеса [Петина, 2006].

Авторы предложили четыре направления оценки эффективности, отвечающие на самые значимые для успешной деятельности компании вопросы: финансы, рынок, на котором 
действуют компании, внутренние бизнес-процессы, а также стремление компании к дальнейшему развитию. Разработанные авторами перспективы, отражающие вышеуказанные аспекты деятельности, были названы соответственно «Финансы», «Клиенты», «Бизнеспроцессы» и «Обучение и рост». Имея внутреннюю иерархию, данные перспективы играют практически одинаковую роль, по мнению авторов, с точки зрения повышения эффективности деятельности компании. При этом необходимо отметить, что основу развития первых трех перспектив обеспечивает наличие в компании стремления $к$ формированию собственного конкурентоспособного интеллектуального капитала, представленного в перспективе «Обучение и рост». Формируя новую и, очевидно, правильную концепцию в условиях новой экономики, предложенная модель BSC не отвечает на вопрос, ответ на который может обеспечить лишь единый критерии эффективности деятельности компании: целесообразна ли реализация той или иной стратегии развития компании с позиции максимизации ее добавленной стоимости?

Целью настоящей статьи является обоснование метода оценки добавленной стоимости всего задействованного капитала компании, включая его неосязаемую часть. Разработка этого метода позволит решить ряд указанных выше ограничений и сформирует инструмент для оценки компаний с превалирующей долей интеллектуального капитала. Предлагаемый в статье метод базируется на идее агрегирования добавленной стоимости различных элементов интеллектуального капитала. При этом в основе рассматриваемого подхода лежит метод RAVE $^{\mathrm{TM}}$ (дословно: усилитель стоимости материальных активов) [Strack, Villis, 2002], предложенный в 2002 году консультантами немецкого офиса BCG Райнером Страком и Ульрихом Виллисом.

Концепция RAVE исходит преимущественно из заявленной выше предпосылки о том, что в настоящее время на первый план в современных предприятиях выходит уже не основной капитал, а интеллектуальный, в том числе знания, которыми обладает и которые использует компания в сфере маркетинга, снабжения и развития персонала. Согласно сделанным ранее выводам, по сравнению с доминирующими в конце $\mathrm{XX}$ века концепциями сбалансированная система показателей избавлена от этого недостатка, однако обладает другим, а именно имеет качественный характер. Концепция RAVE ${ }^{\mathrm{TM}}$, базируясь на принципах сбалансированной системы показателей, дает количественную оценку, что упрощает анализ, а также позволяет определить, какой именно фактор вносит больший вклад в создание стоимости компании [Strack, Villis, 2002].

В зависимости от величины компании, а также ее отраслевой принадлежности, типа рыночной структуры, в условиях которой действует предприятие, изменяются ключевые составляющие капитала, производящего добавленную стоимость. При этом сегодня даже на промышленных предприятиях материальный капитал не играет решающей роли. Однако наиболее очевидный акцент на неосязаемые активы, в частности интеллектуальный капитал, сделан в компаниях, действующих в условиях монополистической конкуренции в сфере торговли и услуг. Для торговых компаний затраты на закупку товара составляют наибольшую часть, поэтому выбор поставщика, а также управление продажами являются наиболее важными. Для компаний, работающих в сфере услуг, наиболее значимым является персонал, то есть для них важно определить эффективные рычаги управления человеческими ресурсами. Помимо капитала, материалов и персонала фирма несет издержки, связанные со своими клиентами. Этот фактор особенно важен для компаний с большими маркетинговыми затратами на привлечение и удержание потребителей. Таким образом, в рассматриваемой концепции авторы выделили четыре ключевых направления: человеческий капитал, финансовый капитал, поставщиков и клиентов. Каждый из них приносит какую-то ценность фирме, которую ниже будем называть экономической добавленной стоимостью (EVA).

Помимо влияния на структуру издержек важность персонала, поставщиков и потребителей определяется их вкладом в успех компании. В то время как предложение инвестиционного капитала на финансовом рынке велико, предложение квалифицированной рабочей силы, поставщиков и выгодных клиентов ограничено. Поэтому качество этих 
факторов каждой компании серьезно влияет на ее стоимость и учитывается вместе с издержками в концепции RAVE ${ }^{\mathrm{TM}}$.

Основной целью предложенной концепции является создание интегрированной системы контроля, которая использует принципы концепции EVA, однако при этом управляет не только инвестированным капиталом, но и человеческим - с помощью подхода, названного Workonomics $^{\mathrm{TM}}$; клиентами - с помощью подхода Custonomics ${ }^{\mathrm{TM}}$; и поставщиками - c помощью подхода Supplynomics ${ }^{\mathrm{TM}}$. Все эти подходы имеют количественную оценку и одинаковые единицы измерения - денежные единицы. Рассмотрим каждый их этих подходов подробнее.

\section{Workonomics}

По мнению авторов, аналогично тому как подход EVA позволяет оценить эффект от инвестирования финансового капитала, Workonomics позволяет оценить эффект от задействованного человеческого капитала, используя целый набор количественных, ориентированных на персонал показателей, которые являются подобием существующих финансовых коэффициентов [Strack, Villis, 2002].

Аналогично подсчету EVA или CVA получаем:

$\mathrm{EVA}=(\mathrm{VAP}-\mathrm{ACP})^{*} \mathrm{P}$

где

- VAP (добавленная стоимость на человека) - это отношение общей добавленной стоимость работников к числу работников. По сути, этот показатель может быть интерпретирован как продуктивность работников. Также этот показатель измеряет необходимость таких мероприятий, как качественный отбор и развитие существующего персонала;

- ACP (средние издержки) - это издержки на развитие персонала и оплату труда в расчете на одного человека;

- Р (число работников) - общее число занятых на предприятии.

Несмотря на то что авторы утверждают, что предложенный им подход структурно и содержательно повторит концепцию экономической добавленной стоимости, можно заметить, что показатель, отражающий, согласно модели RAVE, добавленную стоимость человеческого капитала, не учитывает альтернативную стоимость данного ресурса, которая, в сущности, является ключевым отличием концепции учетной и экономической прибыли. Рассмотрим более подробно, что представляет собой экономическая добавленная стоимость. EVA использует определение экономической прибыли, сформулированное в теории экономического дохода Альфреда Маршалла в 1890 году. Рассматривая прибыль, получаемую собственником бизнеса, Маршалл дает следующее определение экономической прибыли: «То, что остается от прибыли после вычитания процентов по привлечению капитала по текущей ставке, может называться прибылью предпринимательской деятельности или управления» [Grant, 2003]. Основное различие между экономической и бухгалтерской прибылью заключается в том, что компания является прибыльной экономически тогда, когда ее выручка не только покрывает производственные и операционные издержки ведения бизнеса, но и обеспечивает требуемый возврат на капитал, инвестированный собственниками. Таким образом, экономическая добавленная стоимость представляет собой прибыль, оставшуюся после вычета всех прямых издержек на привлечение заемного капитала и косвенных издержек на привлечение собственного капитала. Согласно этому определению, EVA перспектив Workonomics, Custonomics и Supplynomics не может называться добавленной экономической стоимостью, поскольку не учитывает косвенных издержек привлечения человеческого капитала, представляя собой некий оставшийся после вычитания из выручки операционных издержек и прямых затрат на привлечение капитала доход.

Для формирования полноценного аналога экономической прибыли от задействованного 
человеческого капитала нам необходимо было бы учесть в ее расчете альтернативную отдачу от данного ресурса, которая может быть получена в случае применения его в другой компании. Понимая, что предложенный авторами вариант расчета отдачи от элементов интеллектуального капитала не решает указанную выше проблему оценки, предположим на данном этапе анализа, что альтернативная отдача от использования указанных ресурсов равна 0, и решим поставленную задачу с учетом этой предпосылки. Данная предпосылка будет распространена на две другие характеристики стоимости интеллектуального капитала: Custonomics и Supplynomics.

Учитывая это, формы уравнения для капитала и человеческих ресурсов одинаковы, то есть мы можем соотнести WACC со средними издержками на человека, ROI - с добавленной стоимостью на человека, а инвестированный капитал - с количеством занятых. Именно поэтому как финансовый капитал, так и работники компании, являясь важными для компании ресурсами, измеряются с помощью единого подхода и интегрированы в единую систему управления стоимостью.

Стоимость может быть увеличена с помощью следующих методов:

- Рост добавленной стоимости на человека (VAP). Это может быть обеспечено ростом производительности труда, сокращением материальных затрат, усовершенствованием процесса управления персоналом и т.д.

- Увеличение количества занятых (Р), то есть рост количества работников, с превышением средней добавленной стоимости над средними издержками на человека. Таким образом, рост персонала при положительном значении средней отдачи на сотрудника ведет к росту стоимости. Однако если отдача отрицательная, сокращение персонала приведет к увеличению прибыли собственников, так как непродуктивные работники сокращают стоимость.

- Сокращение средних издержек (АСР) приведет к увеличению стоимости. Это может быть связано с перемещением производства в районы с более низкой заработной платой либо сокращением издержек, не связанных с заработной платой.

Данный подход может быть также применен для определения позиции компании или ее подразделения среди других компаний. Доказательством полезности применения данной концепции может служить ситуация, когда и финансовый капитал, и количество работников растет, показатели рентабельности инвестиций также увеличиваются, однако добавленная стоимость на человека сокращается. Таким образом, Workonomics позволяет обнаружить неэффективность внутри компании там, где обычный поход к оценке капитала с этим не справляется.

\section{Custonomics}

В подходе Custonomics на первый план выходит капитал, приносимый потребителями. В данном случае EVA выражается только через характеристики, относящиеся к покупателям, и не использует данные финансового капитала или человеческих ресурсов компании.

$\mathrm{EVA}=(\mathrm{VAC}-\mathrm{ACC}) * \mathrm{C}$,

где

- VAC - добавленная стоимость на покупателя;

- ACC - торговые и маркетинговые издержки на покупателя;

- С - число покупателей.

Ценность компании в данном случае может быть создана путем увеличения добавленной стоимости на покупателя, сокращением торговых и маркетинговых издержек либо увеличением числа клиентов за счет специальных программ привлечения и удержания потребителей.

Пользуясь подобным подходом, мы получаем удобный инструмент контроля для многочисленных клиенториентированных компаний (таких, как интернет-компании, розничные торговые сети, телекоммуникационные, страховые компании и банки). Также эту 
концепцию можно использовать для управления компанией, ее подразделением, сегментом покупателей, а в некоторых случаях можно даже руководить решениями отдельных менеджеров по продажам. Концепция Custonomics шире, чем инструмент контроля, поскольку она привносит множество стратегических вопросов в процесс анализа и сегментации потребителей.

\section{Supplynomics}

Кроме рассмотрения человеческих ресурсов и потребителей существует третий подход, рассматривающий поставщиков. Соответствующая концепция в управлении стоимостью носит название Supplynomics.

В данном подходе эффективность будет измеряться добавленной стоимостью на поставщика (VAS), которая сравнивается со средними затратами на данных контрагентов (ACS). Таким образом, добавленная стоимость компании в рамках концепции Supplynomics оценивается следующим образом:

$\mathrm{EVA}=(\mathrm{VAS}-\mathrm{ACS}) * \mathrm{~S}$.

B этой концепции поставщики также могут быть заменены на продукты или продуктовые группы (материалы).

Ценность в данном случае может быть создана увеличением добавленной стоимости на потребителя или поставленный материал; путем снижения средних издержек на поставщика или продуктовую группу, таких как логистические издержки или издержки складирования, а также закупочных цен напрямую или путем увеличения числа поставщиков S (учитывая, что $\mathrm{VAS}>\mathrm{ACS})$.

Подобный подход облегчает оценку компании и стоимости, созданной конкретными поставщиками, продуктами или продуктовыми группами. Supplynomics актуален для компаний с низким уровнем вертикальной интеграции, таких как розничные фирмы или производители потребительских товаров, в которых может быть определена добавленная стоимость продукта или поставщика.

Как неоднократно подчеркивалось выше, одним из достоинств концепции RAVE ${ }^{\mathrm{TM}}$ является то, что она состоит из количественных показателей. В целом концепция может быть использована для анализа компаний или отдельных подразделений (дочерних компаний, продуктовых сегментов, потребительских сегментов и т.д.) Ex post, данный подход позволяет оценить успешность бизнеса, рассчитанный ex ante, oн может быть использован для принятия решений о распределении ресурсов. целом концепция может быть использована для анализа целых компаний или отдельных подразделений (нная стоимость продукта или по

Мы уже говорили о том, что ценность данной концепции выходит за рамки контроля деятельности компании, так как она включает в себя решение ряда стратегических задач. При этом необходимо отметить, что в настоящее время ограничениями для применения рассмотренной концепции являются нерешенные вопросы относительно:

- расчета экономической добавленной стоимости на базе всех ее составляющих (workonomics, custonomics, supplynomics);

- методов оценки всех составляющих средней отдачи или альтернативной стоимости от использования составляющих интеллектуального капитала: кадровых ресурсов, клиентов и поставщиков.

Авторы концепции предлагают выделить, в сущности, одну из ключевых составляющих добавленной стоимости для конкретной компании и оценивать добавленную стоимость на основе ресурса, который объясняет основную часть ценности, создаваемой фирмой. Методы оценки отдачи на задействованные ресурсы основаны на данной предпосылке. Очевидно, что данное ограничение на использование метода сужает сферу его применения до тех компаний, где существенно превалирует один из составляющих интеллектуального капитала. Например, консалтинговые компании, где практически основным ресурсом является кадровый потенциал, или интернет-компании, где практически 
всю добавленную стоимость создают клиенты. При этом необходимо отметить, что большая часть объектов оценки базируется на нескольких составляющих в различных сочетаниях: клиенты и поставщики, финансовый капитал и персонал и т.д. А также снижение роли финансового капитала не приводит к полной потери его влияния на эффективность деятельности компании. Таким образом, актуальным направлением развития концепции RAVE является разработка метода оценки добавленной стоимости компании на основе всех ключевых составляющих материальных и нематериальных активов компании. В случае если возможно было бы определить независимую отдачу и затраты использования каждого из составляющих активов и при этом отсутствовало бы «пересечение» этих показателей, можно было бы предположить, что интегральный показатель экономической добавленной стоимости (Integrated Economic Value Added - IEVA) представляет сумму показателей добавленной ценности каждого из составляющих материальных и неосязаемых активов. Однако на практике это условие не выполняется. В общем случае интегральный показатель представляет собой объединение (как теоретико-множественная операция) составляющих добавленной стоимости человеческих ресурсов $E V A W$, клиентов $E V A C$, поставщиков $E V A S$ и финансового капитала $E V A F$ (рис. 1):

$I E V A=E V A F \cup E V A W \cup E V A C \cup E V A S$.

Таким образом, для расчета предложенного интегрального показателя необходимо определить степень «пересечения» исходных показателей.

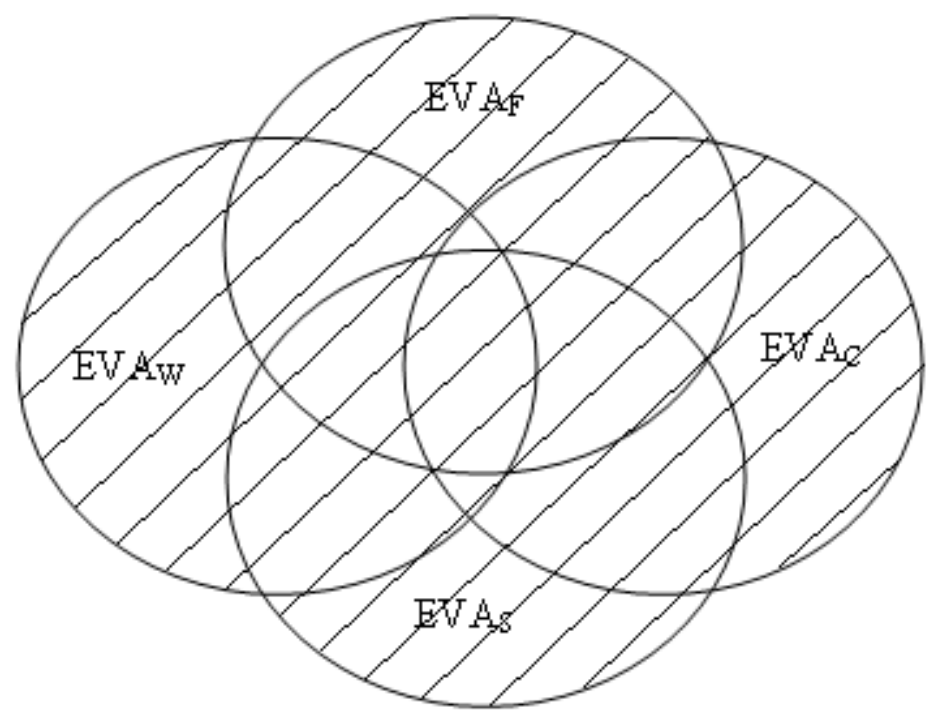

Рисунок 1. Интегральный показатель экономической добавленной стоимости

Кроме того можно предположить, что степень пересечения показателей workonomics, custonomics, supplynomics обуславливаются рядом специфических факторов, в том числе примененной методикой расчета составляющих показателей, размером оцениваемой компании, ее отраслевой принадлежностью, а также характеристикой выбранной стратегии развития компании, в том числе выбранными приоритетами и ключевыми факторами успеха.

Безусловно, описанное развитие подхода RAVE позволяет снять ряд достаточно жестких ограничений, связанных с применением этой модели. Предлагаемый интегральный показатель экономической добавленной стоимости позволяет связать разрозненные индикаторы workonomics, custonomics, supplynomics в единый стратегический показатель эффективности компании. В то же время необходимо отметить, что существующая неопределенность относительно квантификации указанных показателей является серьезным препятствием для предложенного развития модели.

В рамках настоящей статьи произведем попытку оценить интегральный показатель экономической добавленной стоимости, базирующийся на подходе, предложенном консультантами консалтинговой группы BCG. 
Начнем расчет IEVA с определения тех показателей, которые входят в несколько перспектив сразу. При этом следует понимать, что пересечение перспектив концепции определяется не численно, а экономически (то есть одинаковыми по экономическому смыслу элементами затрат или отдачи). Перечислим элементы, входящие в перспективы концепции, для того чтобы далее выделить области пересечения показателей.

\section{Workonomics:}

Пусть МС - это материальные затраты.

$\mathrm{EVA}_{\mathrm{W}}=(\mathrm{VAP}-\mathrm{ACP})^{*} \mathrm{P}=\mathrm{VA}_{\mathrm{E}}-\mathrm{PC}=\mathrm{TR}-\mathrm{MC}-\mathrm{A}-\mathrm{WACC} * \mathrm{IC}-\mathrm{PC}$.

\section{Custonomics:}

Обозначим сумму торговых и маркетинговых издержек как $\mathrm{SMC}_{\Sigma}$. Тогда долю этих издержек, относящуюся к материальным затратам, обозначим как $\mathrm{SMC}_{1}$; относящуюся к амортизации - как $\mathrm{SMC}_{2}$; относящуюся к персональным издержкам - как $\mathrm{SMC}_{3}$; относящуюся к инвестированному капиталу - как $\mathrm{SMC}_{4}$.

$\mathrm{EVA}_{\mathrm{C}}=(\mathrm{VAC}-\mathrm{ACC})^{*} \mathrm{C}=\mathrm{VAC}-\mathrm{SMC}_{\Sigma}=\mathrm{TR}-\mathrm{MC}^{*}-\mathrm{PC}^{*}-\mathrm{A}^{*}-\mathrm{WACC} \cdot \mathrm{IC}^{*}-\mathrm{SMC}_{\Sigma}=$ $\mathrm{TR}-\left(\mathrm{MC}-\mathrm{SMC}_{1}\right)-\left(\mathrm{A}-\mathrm{SMC}_{2}\right)-\left(\mathrm{PC}-\mathrm{SMC}_{3}\right)-\mathrm{WACC}^{*}\left(\mathrm{IC}-\mathrm{SMC}_{4}\right)-\mathrm{SMC}_{\Sigma}$.

\section{Supplynomics:}

Обозначим долю затрат на закупку и хранение материалов, относящуюся к амортизации, как $\mathrm{PFR}_{1}$; относящуюся к персональным издержкам - как $\mathrm{PFR}_{2}$; относящуюся к инвестированному капиталу - как $\mathrm{PFR}_{3}$.

$\mathrm{EVA}_{\mathrm{S}}=(\mathrm{VAS}-\mathrm{ACS}) * \mathrm{~S}=\mathrm{VAS}-\mathrm{MC}=\mathrm{TR}-\mathrm{PC} * *-\mathrm{A}^{* *}-\mathrm{WACC} \cdot \mathrm{IC} * *-\mathrm{MC}=\mathrm{TR}-\mathrm{MC}$ - $\left(\mathrm{A}-\mathrm{PFR}_{1}\right)-\left(\mathrm{PC}-\mathrm{PFR}_{2}\right)-\mathrm{WACC} *\left(\mathrm{IC}-\mathrm{PFR}_{3}\right)$.

\section{Economics:}

$\mathrm{EVA}_{\mathrm{E}}=(\mathrm{ROI}-\mathrm{WACC}) * \mathrm{IC}=\mathrm{NOPAT}-\mathrm{WACC} * \mathrm{IC}=(\mathrm{TR}-$ операционные затраты $)$ * $(1$ -T) $-\mathrm{WACC} * \mathrm{IC}=\mathrm{TR} *(1-\mathrm{T})-$ операционные затраты * $(1-\mathrm{T})-\mathrm{WACC} * \mathrm{IC}$.

Поскольку элементы издержек, вычитаемые из выручки в прочих перспективах, содержатся в операционных затратах, однако явно не выделены, запишем операционные затраты следующим образом:

Операционные затраты $=\mathrm{MC}+\mathrm{PC}+\mathrm{A}+\mathrm{X}$, где $\mathrm{X}-$ операционные затраты, не относящиеся к материальным затратам, издержкам на персонал и амортизации. Тогда:

$\mathrm{EVA}_{\mathrm{E}}=\mathrm{TR} *(1-\mathrm{T})-$ операционные затраты $*(1-\mathrm{T})-\mathrm{WACC} * \mathrm{IC}=\mathrm{TR} *(1-\mathrm{T})-(\mathrm{MC}$ $+\mathrm{PC}+\mathrm{A}+\mathrm{X}) *(1-\mathrm{T})-\mathrm{WACC} * \mathrm{IC}$.

Определение пересечений будет происходить следующим образом. Сначала выделим те элементы, которые находятся на пересечении всех четырех перспектив, рассматриваемых в рамках концепции RAVE. Следующим шагом рассмотрим поочередно области пересечения трех перспектив, затем - попарно области пересечения двух перспектив. После нахождения всех элементов, входящих более чем в одну перспективу, они будут вычтены из итоговой величины экономической добавленной стоимости соответствующей перспективы. Таким образом, в итоге EVA каждой перспективы будет очищена от повторяющихся элементов. Добавив к ним элементы, стоящие на пересечениях перспектив, получим интегральный показатель экономической добавленной стоимости.

Выделим общие для каждой перспективы элементы, вычитаемые из выручки. Как уже отмечалось ранее, важно выделить компоненты добавленной стоимости, несущие одинаковый экономический смысл. Рассмотрим подробнее процесс анализа на примере материальных затрат.

В перспективах Workonomics и Supplynomics из выручки вычитается вся величина материальных издержек. В перспективе Custonomics вычитается величина материальных издержек, скорректированных на долю торговых и маркетинговых издержек. В перспективе Economics учитываются материальные издержки после вычета налогов. Таким образом, нам нужно определить степень пересечения $\mathrm{MC}\left(1\right.$ - Т) и $\left(\mathrm{MC}-\mathrm{SMC}_{1}\right)$ (рис. 2): 


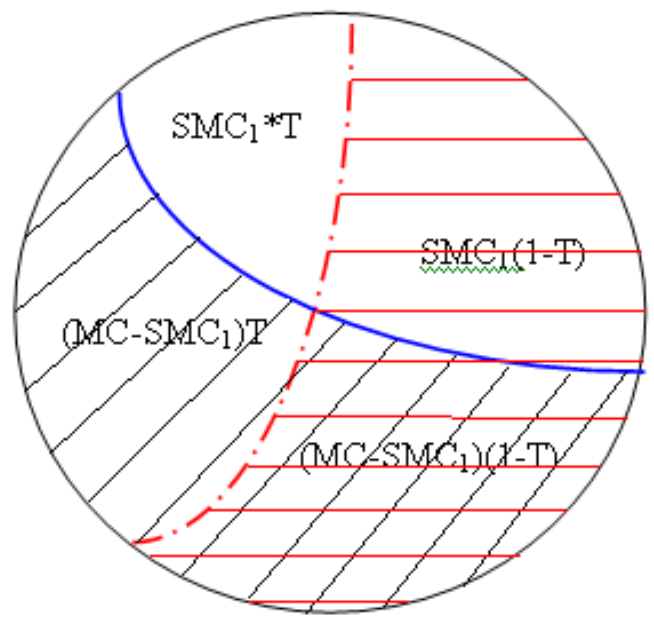

Рисунок 2. Элементы материальных издержек

Пусть вся окружность на рисунке 2 представляет собой материальные издержки. Часть материальных издержек, лежащая выше синей дуги, - это $\mathrm{SMC}_{1}$, часть материальных издержек, лежащая выше красной дуги, - это $\mathrm{MC} * \mathrm{~T}$. Она пересекает $\mathrm{SMC}_{1}$, так как умножение $\mathrm{MC}$ на ставку налога предполагает, что $\mathrm{SMC}_{1}$, как часть материальных издержек, также умножается на эту ставку. Тогда по рисунку видно, что пересечением двух множеств будет являться $\left(\mathrm{MC}-\mathrm{SMC}_{1}\right)(1-\mathrm{T})$.

Теперь рассмотрим амортизацию. В этом случае проблема определения области пересечения усложняется тем, что помимо налогов и торговых и маркетинговых издержек в амортизацию входит часть издержек на закупку и хранение материалов. В перспективе Workonomics из выручки вычитается вся величина амортизации. В перспективе Supplynomics вычитается величина амортизации, скорректированная на часть издержек по закупке и хранению материалов. В перспективе Custonomics вычитается величина амортизации, скорректированная на долю торговых и маркетинговых издержек. В перспективе Economics учитывается амортизация после вычета налогов.

Определим область пересечения четырех множеств с помощью рисунка 3:
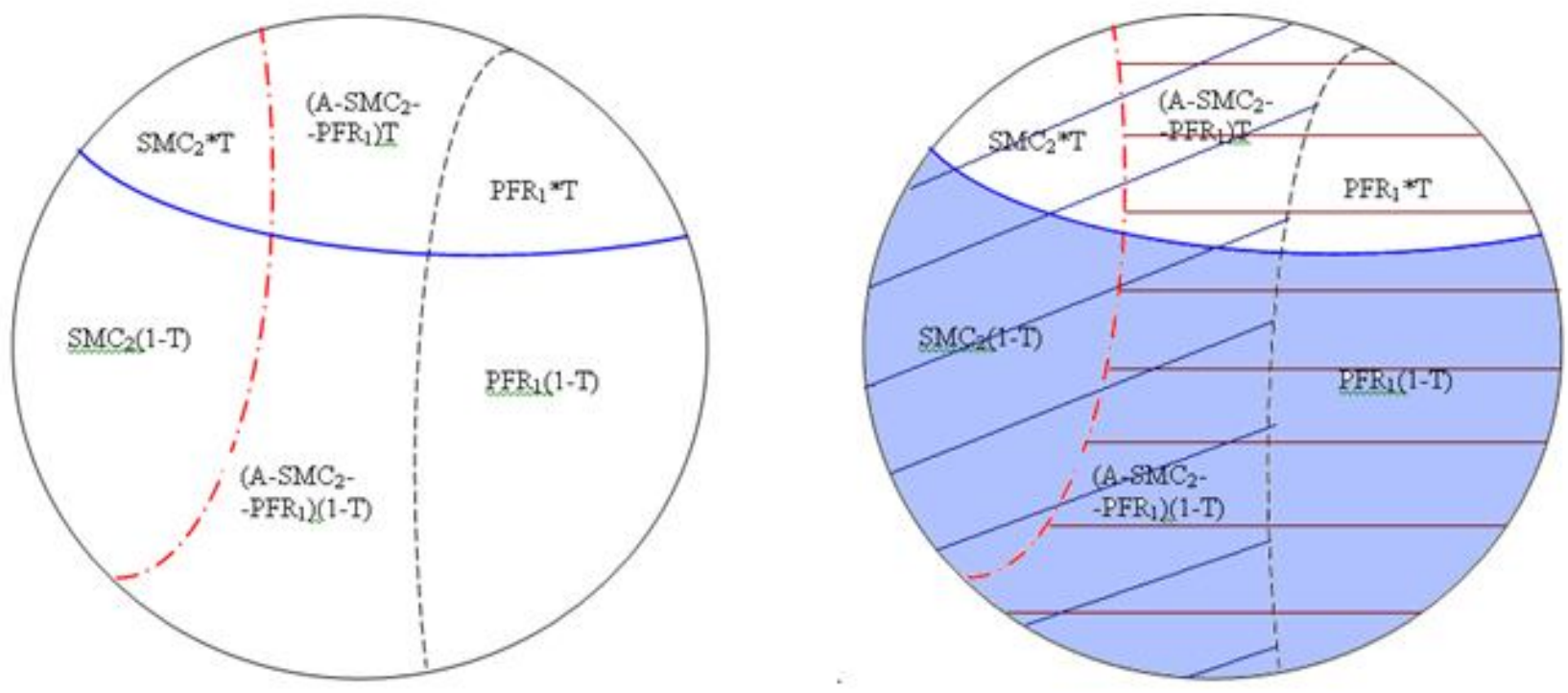

Рисунок 3. Элементы амортизации 
Пусть вся окружность как в правой, так и в левой части рисунка 3 представляет собой амортизацию. Часть амортизации, лежащая выше синей сплошной дуги, - это $\mathrm{A} * \mathrm{~T}$, часть амортизации, лежащая выше красной дуги, нарисованной штрих-пунктиром, - это $\mathrm{SMC}_{2}$, часть амортизации, лежащая выше пунктирной линии, - это $\mathrm{PFR}_{1} . \mathrm{SMC}_{2}$ и $\mathrm{PFR}_{1}$ являются частью амортизации и не пересекаются, исходя из их экономического смысла. Множество А * T пересекает $\mathrm{SMC}_{2}$ и $\mathrm{PFR}_{1}$, так как умножение A на ставку налога предполагает, что все ее компоненты также умножаются на эту ставку. В правой части рисунка 3 с помощью штриховки определяем компоненты амортизации, относящиеся к той или иной перспективе. В итоге найдено пересечение всех четырех множеств: $\left(\mathrm{A}-\mathrm{SMC}_{2}-\mathrm{PFR}_{1}\right)(1$ - T).

Аналогично этим примерам определим пересечение для прочих затрат. Учтем, что на пересечении всех перспектив концепции находится величина посленалоговой выручки, и получим следующие результаты:

$$
\begin{aligned}
& \text { - }\left(\mathrm{MC}-\mathrm{SMC}_{1}\right)(1-\mathrm{T}) \\
& \text { - }\left(\mathrm{A}-\mathrm{SMC}_{2}-\mathrm{PFR}_{1}\right)(1-\mathrm{T}) \\
& \text { - }\left(\mathrm{PC}-\mathrm{SMC}_{3}-\mathrm{PFR}_{2}\right)(1-\mathrm{T}) \\
& \text { - }\left(\mathrm{IC}-\mathrm{SMC}_{4}-\mathrm{PFR}_{3}\right) * \mathrm{WACC} \\
& +\mathrm{TR} *(1-\mathrm{T}) \\
& \text { Пусть }(1)+(2)+(3)+(4)+(5)=\mathrm{G} .
\end{aligned}
$$

Определим, какие элементы экономической добавленной стоимости лежат внутри каждой перспективы помимо компонентов, описываемых выражениями (1), (2), (3), (4), (5). Для этого выделим эти элементы внутри соответствующих слагаемых EVA $, \mathrm{EVA}_{\mathrm{W}}, \mathrm{EVA}_{\mathrm{C}}$, EVAs.

Начнем с рассмотрения перспективы Economics:

$-\mathrm{MC}(1-\mathrm{T})=-\left(\mathrm{MC}-\mathrm{SMC}_{1}\right)(1-\mathrm{T})-\mathrm{SMC}_{1}(1-\mathrm{T})$

$-\mathrm{A}(1-\mathrm{T})=-\left(\mathrm{A}-\mathrm{SMC}_{2}-\mathrm{PFR}_{1}\right)(1-\mathrm{T})-\mathrm{SMC}_{2}(1-\mathrm{T})-\mathrm{PFR}_{1}(1-\mathrm{T})$

$-\mathrm{PC}(1-\mathrm{T})=-\left(\mathrm{PC}-\mathrm{SMC}_{3}-\mathrm{PFR}_{2}\right)(1-\mathrm{T})-\mathrm{SMC}_{3}(1-\mathrm{T})-\mathrm{PFR}_{2}(1-\mathrm{T})$

$-\mathrm{WACC} * \mathrm{IC}=-\left(\mathrm{IC}-\mathrm{SMC}_{4}-\mathrm{PFR}_{3}\right) * \mathrm{WACC}-\mathrm{WACC}\left(\mathrm{SMC}_{4}+\mathrm{PFR}_{3}\right)$

Исходя из этого получим:

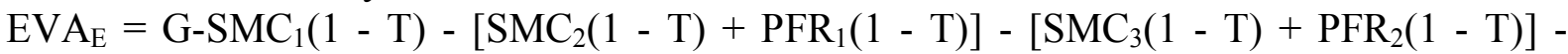
$\mathrm{WACC}\left(\mathrm{SMC}_{4}+\mathrm{PFR}_{3}\right)-\mathrm{X}(1-\mathrm{T})$

Проведя действия, аналогичные преобразованиям выражений перспективы Еconomics, приведенным выше, для остальных трех перспектив, получим:

$\mathrm{EVA}_{\mathrm{W}}=\mathrm{TR} * \mathrm{~T}+\mathrm{G}-\left[\mathrm{MC} * \mathrm{~T}+(1-\mathrm{T}) \mathrm{SMC}_{1}\right]-\left[\mathrm{A} * \mathrm{~T}+\mathrm{SMC}_{2}(1-\mathrm{T})+\mathrm{PFR}_{1}(1-\mathrm{T})\right]-[\mathrm{PC}$ $\left.* \mathrm{~T}+\mathrm{SMC}_{3}(1-\mathrm{T})+\mathrm{PFR}_{2}(1-\mathrm{T})\right]-\left[\mathrm{WACC}\left(\mathrm{SMC}_{4}+\mathrm{PFR}_{3}\right)\right]$

$\mathrm{EVA}_{\mathrm{C}}=\mathrm{TR} * \mathrm{~T}+\mathrm{G}-\left(\mathrm{MC}-\mathrm{SMC}_{1}\right) \mathrm{T}-\left[\left(\mathrm{A}-\mathrm{SMC}_{2}\right) \mathrm{T}+\mathrm{PFR}_{1}(1-\mathrm{T})\right]-\left[\left(\mathrm{PC}-\mathrm{SMC}_{3}\right) * \mathrm{~T}+\right.$ $\left.\mathrm{PFR}_{2}(1-\mathrm{T})\right]-\mathrm{WACC} * \mathrm{PFR}_{3}-\mathrm{SMC}_{\Sigma}$

$\mathrm{EVA}_{\mathrm{S}}=\mathrm{TR} * \mathrm{~T}+\mathrm{G}-\left[\mathrm{MC} * \mathrm{~T}+\mathrm{SMC}_{1}(1-\mathrm{T})\right]-\left[\left(\mathrm{A}-\mathrm{PFR}_{1}\right) \mathrm{T}+\mathrm{SMC}_{2}(1-\mathrm{T})\right]-\left[\left(\mathrm{PC}-\mathrm{PFR}_{2}\right)\right.$ $\left.* \mathrm{~T}+\mathrm{SMC}_{3}(1-\mathrm{T})\right]-\mathrm{WACC} * \mathrm{SMC}_{4}$

После выделения элементов, стоящих на пересечении всех четырех перспектив (а именно G), определим, какие компоненты экономической добавленной стоимости входят одновременно в три перспективы.

\section{Supplynomics + Workonomics + Custonomics:}

Начнем с рассмотрения материальных затрат. В перспективах Supplynomics и Workonomics вычитается величина материальных затрат, умноженная на ставку налога, тогда как в перспективе Custonomics - величина материальных затрат, скорректированная на долю торговых и маркетинговых издержек, умноженная на налоговую ставку. Эти две области пересекаются на величину (MC - $\left.\mathrm{SMC}_{1}\right)$ * T (см. рис. 2).

Рассуждая аналогичным образом относительно амортизации и издержек на персонал, а также пользуясь рисунком 3, получим, что область пересечения этих трех перспектив содержит следующие компоненты: 
$-\left(\mathrm{MC}-\mathrm{SMC}_{1}\right) * \mathrm{~T}$

$-\left(\mathrm{A}-\mathrm{PFR}_{1}-\mathrm{SMC}_{2}\right) * \mathrm{~T}$

$-\left(\mathrm{PC}-\mathrm{PFR}_{2}-\mathrm{SMC}_{3}\right) * \mathrm{~T}$

$+\mathrm{TR} * \mathrm{~T}$

Помимо элементов, находящихся на пересечении всех четырех перспектив (G), а также указанных выше $((6)+(7)+(8)+(9))$, рассматриваемые перспективы содержат следующие слагаемые:

Workonomics: $-\mathrm{SMC}_{1}-\mathrm{SMC}_{2}-\mathrm{SMC}_{3}-\mathrm{T} * \mathrm{PFR}_{1}-\mathrm{T} * \mathrm{PFR}_{2}-\mathrm{WACC} *\left(\mathrm{SMC}_{4}+\mathrm{PFR}_{3}\right)$

Custonomics: $-\mathrm{SMC}_{\Sigma}-\mathrm{T} * \mathrm{PFR}_{1}-\mathrm{T} * \mathrm{PFR}_{2}-\mathrm{WACC} * \mathrm{PFR}_{3}$

Supplynomics: $-\mathrm{SMC}_{1}-\mathrm{SMC}_{2}-\mathrm{SMC}_{3}-\mathrm{WACC} * \mathrm{SMC}_{4}$

Economics + Custonomics + Supplynomics:

Рассматриваемые перспективы не имеют общих элементов.

\section{Economics+Custonomics+Workonomics:}

Область пересечения содержит один элемент издержек:

$-\mathrm{WACC} * \mathrm{PFR}_{3}$

\section{Economics + Supplynomics + Workonomics:}

Область пересечения рассматриваемых перспектив содержит следующие издержки:

$-\left(1-\mathrm{T}^{-} \mathrm{SMC}_{1}\right.$

$-(1-\mathrm{T}) \mathrm{SMC}_{2}$

$-(1-\mathrm{T}) \mathrm{SMC}_{3}$

$-\mathrm{WACC} * \mathrm{SMC}_{4}$

После вычитания всех выделенных ранее и в данном пункте элементов, находящихся на пересечении, перспективы содержат следующие слагаемые:

Economics: $-(1-\mathrm{T}) * \mathrm{PFR}_{1}-(1-\mathrm{T}) * \mathrm{PFR}_{2}-\mathrm{X} *(1-\mathrm{T})$

Custonomics: $-\mathrm{SMC}_{\Sigma}-\mathrm{T} * \mathrm{PFR}_{1}-\mathrm{T} * \mathrm{PFR}_{2}$

Workonomics: $-\left(\mathrm{SMC}_{1}+\mathrm{SMC}_{2}+\mathrm{SMC}_{3}\right) * \mathrm{~T}-\mathrm{T} * \mathrm{PFR}_{1}-\mathrm{T} * \mathrm{PFR}_{2}$

Supplynomics: $-\left(\mathrm{SMC}_{1}+\mathrm{SMC}_{2}+\mathrm{SMC}_{3}\right) * \mathrm{~T}$

На следующем этапе определим элементы, находящиеся на пересечении двух перспектив. Исходя из набора нераспределенных элементов, перечисленных выше, можно заметить, что иметь общие элементы будут следующие перспективы:

Workonomics + Supplynomics: $-\left(\mathrm{SMC}_{1}+\mathrm{SMC}_{2}+\mathrm{SMC}_{3}\right) \mathrm{T}$

Economics + Custonomics: $-\operatorname{SMC}_{\Sigma}(1-\mathrm{T})$

Workonomics + Custonomics: $-\mathrm{T} * \mathrm{PFR}_{1}-\mathrm{T} * \mathrm{PFR}_{2}$

После вычитания всех выделенных ранее элементов, находящихся на пересечениях нескольких множеств ((1) - (16)), найдем компоненты затрат, относящиеся только к одной перспективе.

Economics: $-(1-\mathrm{T}) * \mathrm{PFR}_{1}-(1-\mathrm{T}) * \mathrm{PFR}_{2}-\left(\mathrm{X}-\mathrm{SMC}_{\Sigma}\right) *(1-\mathrm{T})$

Custonomics: $-\mathrm{SMC}_{\Sigma} * \mathrm{~T}$

Теперь, после того, как были определены элементы, входящие сразу в несколько перспектив, можем рассчитать показатель IEVA. Для этого сложим все элементы, входящие в пересечения двух и более перспектив (1) - (17) и прибавим к ним элементы, относящиеся только к одной перспективе (18) и (19).

$\mathrm{IEVA}=\mathrm{TR} *(1-\mathrm{T})-\left(\mathrm{MC}-\mathrm{SMC}_{1}\right)(1-\mathrm{T})-\left(\mathrm{A}-\mathrm{SMC}_{2}-\mathrm{PFR}_{1}\right)(1-\mathrm{T})-\left(\mathrm{PC}-\mathrm{SMC}_{3}-\right.$ $\left.\mathrm{PFR}_{2}\right)(1-\mathrm{T})-\left(\mathrm{IC}-\mathrm{SMC}_{4}-\mathrm{PFR}_{3}\right) * \mathrm{WACC}+\mathrm{TR} * \mathrm{~T}-\left(\mathrm{MC}-\mathrm{SMC}_{1}\right) * \mathrm{~T}-\left(\mathrm{A}-\mathrm{PFR}_{1}-\mathrm{SMC}_{2}\right) * \mathrm{~T}-$ $\left(\mathrm{PC}-\mathrm{PFR}_{2}-\mathrm{SMC}_{3}\right) * \mathrm{~T}-\mathrm{WACC} * \mathrm{PFR}_{3}-(1-\mathrm{T}) \mathrm{SMC}_{1}-(1-\mathrm{T}) \mathrm{SMC}_{2}-(1-\mathrm{T}) \mathrm{SMC}_{3}-\mathrm{WACC} *$ $\mathrm{SMC}_{4}-\left(\mathrm{SMC}_{1}+\mathrm{SMC}_{2}+\mathrm{SMC}_{3}\right) \mathrm{T}-\mathrm{SMC}_{\Sigma}(1-\mathrm{T})-\mathrm{T} * \mathrm{PFR}_{1}-\mathrm{T} * \mathrm{PFR}_{2}-(1-\mathrm{T}) * \mathrm{PFR}_{1}-(1-\mathrm{T}) *$ $\mathrm{PFR}_{2}-\left(\mathrm{X}-\mathrm{SMC}_{\Sigma}\right) *(1-\mathrm{T})-\mathrm{SMC}_{\Sigma} * \mathrm{~T}=\mathrm{TR}-\mathrm{MC}-\mathrm{A}-\mathrm{PC}-\mathrm{IC} * \mathrm{WACC}-\mathrm{SMC}_{\Sigma} * \mathrm{~T}-\mathrm{X} *(1-$ 
$\mathrm{T})$

Полученная формула позволяет рассчитать интегральный показатель экономической добавленной стоимости, который представляет собой сумму добавленных стоимостей, генерируемых всеми материальными и нематериальными активами компании, очищенную от повторяющихся элементов. Расчеты производились на основании метода расчета экономической добавленной стоимости, создаваемой материальными активами, поставщиками, клиентами и сотрудниками компании, предложенного создателями концепции RAVE Страком и Виллисом.

Можно предположить, что степень пересечения показателей перспектив Еconomics, Workonomics, Custonomics, Supplynomics неодинакова для различных компаний и обуславливается рядом специфических факторов, в частности размером оцениваемой компании, ее отраслевой принадлежностью, выбранной стратегией развития, в том числе приоритетами и ключевыми факторами успеха. Для того чтобы проверить это предположение, применим полученную выше формулу расчета интегрального показателя экономической добавленной стоимости к компаниям разных сфер деятельности: консалтинговой компании «ИПЭБИ», торговой компании ООО «Трейд» и ЗАО «Фотон», относящейся к сфере производства.

Расчет общей добавленной стоимости, генерируемой всеми активами ООО «ИПЭБИ», ООО «Трейд» и $3 \mathrm{AO}$ «Фотон», приведен в таблице 1.

Таблица 1

Расчет простого и интегрального показателей экономической добавленной стоимости ООО «ИПЭБИ», ООО «Трейд» и ЗАО «Фотон» в 2009 г., тыс.руб.

\begin{tabular}{|l|l|l|l|}
\hline & ЗАО «Фотон» & $\begin{array}{c}\text { ООО } \\
\text { «ПЭБИ» }\end{array}$ & ООО «Трейд» \\
\hline Выручка & 57429 & 725,05 & 366980 \\
\hline Материальные издержки & 25375,75 & 0,00 & 146786,00 \\
\hline Амортизация & 1305,34 & 0,00 & 1960,00 \\
\hline Издержки на персонал & 16600,79 & 396,53 & 13453,00 \\
\hline Инвестированный капитал & 24407 & 500 & 131875 \\
\hline $\mathrm{WACC}_{\mathbf{1}} \%$ & 21 & 26 & 20,10 \\
\hline $\mathrm{PFR}_{1}$ & 0 & 0 & 0 \\
\hline $\mathrm{PFR}_{2}$ & 47,7 & 0 & 0 \\
\hline $\mathrm{PFR}_{3}$ & 1696 & 0 & 21340 \\
\hline $\mathrm{X}$ & 12389,12 & 123,5 & 170214,00 \\
\hline $\mathrm{T}$ & 0,29 & 0,21 & 0,14 \\
\hline EVA & $\mathbf{- 3 8 7 7 , 4 7}$ & $\mathbf{3 1 , 5 2}$ & $\mathbf{3 0 2 7 , 5}$ \\
\hline IEVA & $\mathbf{4 9 , 8 4}$ & $\mathbf{1 0 0 , 4 8}$ & $\mathbf{3 1 ~ 6 4 7 , 0 7}$ \\
\hline
\end{tabular}

Материальные и нематериальные активы всех анализируемых компаний создают в 2009 году положительную общую экономическую добавленную стоимость. Полученное значение превышает экономическую добавленную стоимость за счет добавленной стоимости, создаваемой человеческим капиталом компании - поставщиками, клиентами и сотрудниками. За счет того что показатель IEVA очищен от повторяющихся элементов, он не равен сумме экономических добавленных стоимостей четырех перспектив концепции RAVE.

Наибольшую общую экономическую добавленную стоимость генерирует ООО «Трейд», тогда как показатели IEVA 3АО «Фотон» и ООО «ИПЭБИ» лишь незначительно отличаются от нуля. Это говорит о возможности снижения величины показателя до отрицательной величины в следующих периодах. Однако высокое значение интегрального показателя торговой компании может объясняться эффектом размера. Для того чтобы 
провести более корректное сравнение, нормализуем интегральный показатель экономической добавленной стоимости каждой компании на балансовую стоимость ее активов (табл. 2.):

Таблица 2

Интегральные показатели экономической добавленной стоимости ООО «ИПЭБИ», ООО «Трейд» и ЗАО «Фотон», нормированные на величину балансовой стоимости активов,

\begin{tabular}{|l|l|}
\hline & IEVA/BVA \\
\hline ООО «ИПЭБИ» & 0,20 \\
\hline ЗАО «Фотон» & 0,0018 \\
\hline ООО «Трейд» & 0,24 \\
\hline
\end{tabular}

После исключения влияния эффекта размера компании на ее интегральный показатель были получены несколько иные результаты. Материальные и нематериальные активы ООО «Трейд» по-прежнему генерируют наибольшую добавленную стоимость, однако исходя из нормализованных данных активы ООО «ИПЭБИ» задействованы практически с такой же эффективностью. Результаты деятельности ЗАО «Фотон» по-прежнему находятся на самом низком уровне (в 130 раз хуже показателя ООО «Трейд»).

Рассмотрим, в какой степени пересекаются перспективы концепции RAVE, рассчитанные на основании данных анализируемых компаний (табл. 3) Так как положительную часть пересечений составляет только выручка, рассмотрим отрицательные элементы.

Таблица 3

Величина отрицательных элементов области пересечения перспектив концепции RAVE для ООО «ИПЭБИ», ООО «Трейд» и ЗАО «Фотон», \%

\begin{tabular}{|l|l|l|l|}
\hline \multicolumn{1}{|c|}{ Пересекающиеся перспективы } & ЗАО «Фотон» & $\begin{array}{c}\text { ООО } \\
\text { «ПЭБИ» }\end{array}$ & $\begin{array}{c}\text { ООО } \\
\text { «рейд» }\end{array}$ \\
\hline Bce перспективы & 63,77 & 72,72 & 47,50 \\
\hline Supplynomics+Workonomics+Custonomics & 20,21 & 12,51 & 7,03 \\
\hline Economics+Custonomics+Workonomics & 1,10 & 0,00 & 1,28 \\
\hline Economics+ Supplynomics +Workonomics & 0,39 & 0,19 & 0,47 \\
\hline Workonomics+Supplynomics & 0,00 & 0,00 & 0,00 \\
\hline Economics+Custonomics & 0,70 & 0,41 & 2,00 \\
\hline Workonomics+Custonomics & 0,02 & 0,00 & 0,00 \\
\hline Economics & 13,53 & 14,06 & 41,38 \\
\hline Custonomics & 0,28 & 0,11 & 0,34 \\
\hline
\end{tabular}

Мы видим, что показатели перспектив концепции RAVE, рассчитанные для ЗАO «Фотон», ООО «Трейд» и ООО «ИПЭБИ», компаний, принадлежащих к разным сферам деятельности и имеющим различный размер, пересекаются в разной степени, что позволяет сделать вывод о влиянии типа компании на величину пересечения показателей различных перспектив. Однако для более детальных выводов необходимо рассматривать компании со схожими показателями эффективности перспектив, иначе величина областей пересечения будет определяться помимо типа компании еще и эффективностью задействованных материальных и нематериальных ресурсов.

Подводя итоги приведенного анализа, можно сделать вывод, что использование интегрального показателя экономической добавленной стоимости позволяет расширить возможности анализа и учесть одновременный вклад материальных активов и интеллектуального капитала в создание стоимости. При анализе деятельности ЗАО «Фотон» в рамках концепции EVA было получено отрицательное значение, что говорит о том, что 
компания разрушает стоимость. Если рассмотреть помимо финансовых активов нефинансовые, такие, как сотрудники компании, ее поставщики и клиенты, увидим, что сотрудники и клиенты компании создают стоимость. При этом возникает необходимость оценить общую суммарную добавленную стоимость, создаваемую всеми активами компании. Интегральный показатель экономической добавленной стоимости ЗАО «Фотон» положителен, что говорит о том, что материальные и нематериальные активы компании суммарно генерируют положительную добавленную стоимость. Неэффективное использование капитала и отрицательное значение показателя EVA финансовой перспективы еще не говорит о том, что в целом компания не создает стоимость для собственников.

С помощью интегрального показателя экономической добавленной стоимости легче проводить сравнение эффективности деятельности различных компаний. Концепция RAVE позволяла сравнивать лишь эффективность деятельности компаний в рамках той или иной концепции, не предлагая единого показателя. На основе первоначального анализа экономической добавленной стоимости можно сделать вывод о том, что ООО «ИПЭБИ» наиболее эффективно использует капитал, тогда как ООО «Трейд» - нематериальные активы. При этом невозможно определить, какая из двух компаний генерирует большую общую добавленную экономическую стоимость. Использование интегрального показателя позволило определить, что материальные и нематериальные активы ООО «Трейд» приносят на 20\% большую добавленную стоимость, нежели активы ООО «ИПЭБИ».

Показатель IEVA в дальнейшем может быть использован в рамках предложенной авторами концепции RAVE методологии операционного и финансового управления. С его помощью можно выделить основные факторы стоимости компании, относящиеся как к материальным, так и к нематериальным активам, провести декомпозицию и построить дерево основных факторов стоимости, провести анализ чувствительности и анализ сценариев и построить таблицу главных факторов стоимости компании, с помощью которой в дальнейшем будет осуществляться контроль и управление. Анализ основных факторов стоимости в рамках концепции RAVE позволяет выявить факторы с разбивкой по отдельным перспективам. Интегральный показатель позволяет выделить только те факторы, которые значимы для общей добавленной стоимости компании, не учитывая те, которые могли быть выделены в ходе анализа отдельных перспектив, однако влияние которых на IEVA незначительно. Это позволяет избежать контроля над излишним количеством факторов, не влияющих на общую добавленную стоимость.

Интегральный показатель экономической добавленной стоимости дает дополнительные возможности для анализа. Он дает возможность просчитать альтернативные стратегии развития компании и сравнить общую добавленную стоимость, получаемую в их рамках, с базовым вариантом. К примеру, было бы интересно рассмотреть альтернативную торговую политику для ООО «Трейд». В настоящее время компания устанавливает минимальную наценку на товары для привлечения большего количества покупателей. При увеличении размера наценки компания будет получать большую прибыль в расчете на один товар, однако при этом количество покупателей и размер приобретаемой партии товара сократятся. Оценка изменения интегрального показателя экономической добавленной стоимости позволит оценить, будет ли альтернативная стратегия более выгодной для ООО «Трейд».

Разработка метода расчета интегрального показателя экономической добавленной стоимости проводилась на основе методики расчета стоимости, создаваемой поставщиками, клиентами и сотрудниками, предложенной Страком и Виллисом в рамках концепции RAVE. Методика расчета экономической добавленной стоимости финансовой перспективы определена в концепции EVA, созданной Стюартом и Стерном, добавленные стоимости прочих перспектив было предложено определять аналогично.

Необходимо еще раз отметить, что представленный расчет показателя интегральной экономической добавленной стоимости справедлив для случая выполнения предпосылки о том, что альтернативная стоимость использования ресурсов компании равна 0 . В целях преодоления указанного ограничения актуальным направлением будущего исследования в 
рамках концепции RAVE является разработка метода расчета экономической добавленной стоимости перспектив Workonomics, Custonomics и Supplynomics согласно классическому еe определению, а также последующая разработка метода оценки добавленной стоимости компании на основе всех ключевых составляющих материальных и нематериальных активов компании, аналогично подходу, представленному в настоящей статье.

\section{Список литературы}

1. Петина Н. BSC, EVA, MBO, KPI, Six Cigm, а также ISO, TQM, CMK, Lean manufacturing, TPS, самообучающаяся организация - современные концепции управления: [Электронный ресурс] / Электрон. ст.- Режим доступа http://www.rusconsult.ru/cms-news.php?mode=view_news\&id=545.

2. Редченко К. EVАлюция сбалансированной системы показателей, 2002: [Электронный peсурс ] / Электрон. ст.- Режим доступа: www.cfin.ru/management/controlling/evaluation.shtml.

3. Ehrbar, A. (1998), Stern, Stewart \& Co EVA: The Real Key to Creating Wealth. New York: Wiley\&Sons, Inc., P. 256.

4. Fernandez, P. EVA and Cash Value Added do not measure shareholder value creation / P. Fernandez. University of Navarra: IESE Business School, 2001.

5. Grant, J.L. (2003), Foundations of Economic Value Added, 2nd Edition, New Jersey: Wiley\&Sons, Inc.

6. Stelter, D. (2000), New Perspectives on Value Creators. A Study of the World's Top Performers/ D. Stelter, G.Frazis, G. Hansell, M. Joiner, E. Olsen. The Boston Consulting Group.

7. Strack, R., Villis, U. (2002), RAVE: Integrated Value Management for Customer, Human, Supplier and Invested Capital, European Management Journal, 20(2) 2002 147-158.

8. Value driver tree financials: [Электронный pecypc] / Электрон. ст.- Режим доступа: http://www.obermatt.com/knowledge/value-based-mgmt/value-driver-tree/financials. 